INPLASY

PROTOCOL

To cite: Tang et al.

Remimazolam versus traditional sedatives for procedural sedation: $\mathrm{A}$ systematic review and metaanalysis of efficacy and safety outcomes. Inplasy protocol 202210054. doi:

10.37766/inplasy2022.1.0054

Received: 11 January 2022

Published: 11 January 2022

Corresponding author:

Yun Tang

503621518@qq.com

Author Affiliation:

Department of Critical Care

Medicine, Union Hospital,

Tongji Medical College, Huazhong University of

Science and Technology,

Wuhan, China.

Support: None.

Review Stage at time of this submission: Preliminary searches.

Conflicts of interest:

None declared.

\section{Remimazolam versus traditional sedatives for procedural sedation: A systematic review and meta-analysis of efficacy and safety outcomes}

Tang, Y1; Yang, X2; Yu, Y3; Shu, H4; Xu, J5; Zou, X6; Yuan, S7; Shang, $Y^{8}$.

Review question / Objective: The aim of the present study was to pool data from all available RCTs to systematically compare the efficacy and safety of remimazolam with traditional sedatives for procedural sedation.

Condition being studied: The majority of procedures such as colonoscopy and bronchoscopy are performed with some form of sedation. Remimazolam is a novel and ultra-shortacting benzodiazepine currently developed for procedural sedation. Previous studies suggested that remimazolam might be a reasonable selection in procedural sedation.

Information sources: We will search articles in Cochrane Library, Embase, PubMed, Web of Science and ClinicalTrials.gov. Reference list of all selected articles will independently screened to identify additional studies left out in the initial search.

INPLASY registration number: This protocol was registered with the International Platform of Registered Systematic Review and Meta-Analysis Protocols (INPLASY) on 11 January 2022 and was last updated on 11 January 2022 (registration number INPLASY202210054).

\section{INTRODUCTION}

Review question / Objective: The aim of the present study was to pool data from all available RCTs to systematically compare the efficacy and safety of remimazolam with traditional sedatives for procedural sedation.

Condition being studied: The majority of procedures such as colonoscopy and bronchoscopy are performed with some form of sedation. Remimazolam is a novel 
and ultra-short-acting benzodiazepine currently developed for procedural sedation. Previous studies suggested that remimazolam might be a reasonable selection in procedural sedation.

\section{METHODS}

Participant or population: Adults receiving procedural sedation will be included.

Intervention: Remimazolam is the main intervention.

Comparator: Traditional sedatives are comparators.

Study designs to be included: Randomized controlled trials (RCTs) will be included.

Eligibility criteria: Case reports, reviews, expert opinions, abstracts, and duplicate publications were excluded.

Information sources: We will search articles in Cochrane Library, Embase, PubMed, Web of Science and ClinicalTrials.gov. Reference list of all selected articles will independently screened to identify additional studies left out in the initial search.

Main outcome(s): Procedure success rate.

Quality assessment / Risk of bias analysis: The methodological quality of included studies will be assessed using the Cochrane Risk of Bias Tool.

Strategy of data synthesis: Results will be analyzed using Review Manager 5.3 by the Cochrane Collaboration. We will use the $\mathbf{I}^{2}$ statistic to assess the statistical heterogeneity among included studies. If significant heterogeneity exist, we will carry out sensitivity analyses or subgroup analyses to evaluate the statistical stability of our findings.

Subgroup analysis: The traditional sedative agents group will be divided into midazolam group and propofol group to conduct subgroup analysis.
Sensitivity analysis: Where single studies appeared to contribute excessively to heterogeneity, we performed sensitivity analyses using the leave-one-out method to test the robustness of our findings after exclusion of these studies.

Country(ies) involved: China.

Keywords: remimazolam, midazolam, propofol, procedural sedation, metaanalysis.

Contributions of each author:

Author 1 - Yun Tang.

Author 2 - Xiaobo Yang.

Author 3 - Yuan Yu.

Author 4 - Huaqing Shu.

Author 5 - Jiqian Xu.

Author 6 - Xiaojing Zou.

Author 7 - Shiying Yuan.

Author 8 - You Shang. 